\title{
El Dorado de Wanli: La embajada imperial a las Filipinas de $1603^{*}$
}

\author{
The Gold of Wanli: The Imperial Embassy \\ to the Philippines in 1603
}

\author{
Chenguang $\mathrm{Li}$ \\ ORCID iD: https://orcid.org/0000-0002-4829-3354 \\ Universidad de Estudios Internacionales de Zhejiang, \\ Hangzhou, China \\ Jesús Paniagua Pérez \\ ORCID iD: https://orcid.org/0000-0002-4356-6229 \\ Universidad de León, España
}

En este trabajo, apoyándonos en las fuentes primarias tanto en español como en chino, intentamos recuperar aspectos cruciales de la embajada que el emperador Wanli, de la dinastía Ming, envió a las Filipinas para comprobar la existencia de yacimientos de oro en las cercanías de Cavite. Así, daremos un papel relevante tanto a los protagonistas como al proceso de un asunto que tenía una gran importancia para la economía china de la época y que marcó las tempranas relaciones entre los dos imperios en los inicios del siglo XVII.

Palabras Clave: Dinastía Ming; Wanli; Filipinas; China; diplomacia; embajada; oro; Sangley.

In this paper, based on the primary sources in both Spanish and Chinese, we attempt to recover crucial aspects of the embassy sent by the Emperor Wanli of the Ming Dynasty to the Philippines to verify the existence of deposits of gold in the vicinity of Cavite. We shall thus stress the significance of both the protagonists and the process of an event that had a great importance for the Chinese economy of the time, which marked the early relations between these two empires at the start of the $17^{\text {th }}$ century.

KeYwords: Ming Dynasty; Wanli; Philippines; China; Diplomacy; Embassy; Gold; Sangley.

Copyright: (C) 2021 CSIC. Este es un artículo de acceso abierto distribuido bajo los términos de la licencia de uso y distribución Creative Commons Reconocimiento 4.0 Internacional (CC BY 4.0).

* El presente estudio se ha realizado en el marco del Proyecto de Investigación «Recopilación, traducción e investigación sobre los archivos diplomáticos relativos a China durante los siglos XVI y XIX en España» (19VJX042), financiado por la Oficina Nacional de Filosofía y Ciencias Sociales de China. 


\section{Introducción}

El encuentro entre los dos imperios más poderosos del siglo XVI, el español de la Casa de Habsburgo y el chino de la dinastía Ming, fue una consecuencia inevitable de la conquista española de Filipinas, que dio paso a los contactos directos con la mítica China, especialmente a partir de 1593, a través de la base comercial que se estableció en Manila, aunque las relaciones entre ambos espacios venían de muy antiguo. La intensificación que se produjo en la segunda mitad del siglo XVI tuvo mucho que ver con la puesta en marcha del Galeón de Manila (1565), que ponía en circulación hacia América y Europa los productos orientales, y hacia China la plata americana.

La consecuencia inmediata de aquella relación comercial fue la emigración masiva de los habitantes de la provincia de Fujian a Filipinas a partir de los años setenta del siglo XVI. ${ }^{1}$ Dicha comunidad, en su afán por mantener una convivencia pacífica, desde los primeros años mostró su respeto hacia las disposiciones de las autoridades españolas. No obstante, aquella coexistencia no evitó los conflictos entre ambas comunidades, que recelaban entre sí, como lo prueba el tema que nos ocupa. Aquella desconfianza había tenido otros antecedentes, como el del 25 de octubre de 1593, que costó la vida al propio gobernador español, Gómez Pérez de Dasmariñas, cuando se desplazaban a la conquista de las Molucas, dando lugar a una búsqueda infructuosa de los asesinos por la costa china. ${ }^{2} \mathrm{El}$ temor aumentó en 1594 con una falsa alarma, cuando varios navíos chinos con soldados se dejaron ver en Filipinas, aunque su finalidad no era un ataque, sino la captura de habitantes del imperio que habían salido sin licencia.

Aquella desconfianza que se iba instalando empeoró al llegar a Manila la embajada enviada por el emperador Wanli (1573-1620), consecuencia de una proposición fantasiosa del sangley Zhang Zhi para adueñarse

1 Al citar los textos en chino, se utiliza el sistema «Pin yin» —romanización del idioma chino-. Es más, traducimos del chino al español los títulos de las fuentes, nombres de los autores, etc. Sin embargo, por lo que se refiere a los términos y a los topónimos lexicalizados en español, los citamos según las normas de la Academia de la Lengua. A título de ejemplo, en el caso de la capital de la dinastía Ming y también de la presente China, en vez de Beijing, utilizaremos Pekín. He aquí algunos lugares que aparecen frecuentemente en el presente trabajo: Fujian, que también se registra en la bibliografía española como Hoquien, Fukién, etc.; la capital de Fujian, Fuzhou, puede parecer como Hochiu, Hochou, etc.; la ciudad costera de Zhangzhou, desde donde salían la mayoría de los sangleyes en los siglos XVI y XVII se transcribe como Chincheo; la ciudad Quanzhou se denomina en las fuentes occidentales como Zaitón, Zeiton, Zaitun, etc. Véase Ollé Rodríguez, 2002, 243-271; Liao y Hui, 2013; Li, 2018.

2 Fish, 2011, 126. 
de una especie de Dorado oriental, próximo a Cavite, que es el objeto de este artículo y que daría lugar al primer levantamiento de los sangleyes, en 1603 , que costó la vida a la casi totalidad de la primera generación de los inmigrantes. $^{3}$

\section{El encuentro entre dos imperios en las Filipinas}

Los contactos entre China y las Filipinas estaban en relación directa con su proximidad. El primer chino de nombre conocido en la isla fue Zheng Guoxi, natural de Fujian, cuya lapida funeraria en Leyte data del año $661 .{ }^{4}$ Además, las relaciones económicas están documentadas a partir del siglo X, cuando los comerciantes de Mait, en Mindoro, llegaron a Cantón en 982, durante el reinado de la dinastía Song (960-1127). ${ }^{5}$ Igualmente, las descripciones de Zhao Rushi en su obra Zhu fan zhi (1225) hacen referencia a las actividades comerciales de los habitantes de Fujian. ${ }^{6}$ Por tanto, como precedentes del tema que nos ocupa, debemos tener en cuenta algunas consideraciones fundamentales.

En primer lugar, que con el acceso al trono imperial de la dinastía Ming (1368-1644), la relación entre China y el sureste asiático se intensificó, como lo prueba la Ming shi (Historia de Ming), ${ }^{7}$ que menciona la

3 Para más información de la matanza de los chinos en las Filipinas en 1603, véase Borao, 1998b, 233-234.

4 Yang y Ye, 1993, 13.

5 Song shi (Historia de Song), vol. 489. Se trata de una obra que contiene información de todo tipo acerca de la dinastía. Editar y recopilar la historia general sobre la dinastía anterior por la posterior fue una tradición milenaria en la historia china, con el fin de resumir lo que había pasado y evitar los errores que se habían cometido por los mandatorios precedentes. Song shi fue redactada por la dinastía Yuan (1271-1368) entre 1343 y 1345, y se entregó a la Corte en 1346. Se halla Song shi íntegramente transcrita en «Zhong guo zhe xue shu dian zi hua ji hua (Plan de obras filosóficas electrónicas de China)», disponible en: https://ctext.org/wiki.pl?if=en\&res=975976 [Consultado: 10/01/2021].

6 Zhao, Rushi (1170-1231) fue un miembro de la familia real de la dinastía Song del Sur (1127-1279). Escribió una obra de dos volúmenes para describir los países extranjeros ya conocidos hasta su época y las relaciones que mantenían con China. Lo hizo durante los años que estuvo al cargo del «Shibosi» de Fujian, la institución dedicada exclusivamente a gestionar los asuntos vinculados con el sistema tributario de la China antigua, tales como recibir a los enviados de los países vasallos, gestionar sus peticiones, etc. En cuanto a la mencionada obra y a las descripciones sobre los chinos en las Filipinas, véase Feng, 2000 [1940], 80-82.

7 Ming shi, en español Historia de Ming, se trata de una obra que contiene información de todo tipo acerca de dicha dinastía. Fue editada durante la dinastía Qing (1636-1912) entre los siglos XVII y XVIII, y se entregó a la Corte en 1739. Ming shi contiene en total cuatro partes en 336 tomos. Entre ellos, del $1 .^{\circ}$ al $24 .^{\circ}$ constituyen las biografías de cada mandatario de la Ming. Los tomos del $25 .^{\circ}$ al 99. ${ }^{\circ}$ incluyen informaciones variadas, por ejemplo, la geografía, la demografía, las infraestructuras, etc. 
misión enviada en 1327 por el gobernador de Luzón; incluso sabemos de otras dos que tuvieron lugar en 1405 y 1410 respectivamente. Con la presencia española en la zona aquellas relaciones se fomentaron, especialmente las económicas. Ya con Legazpi, en 1572, comenzó la arribada masiva de comerciantes chinos, ${ }^{8}$ por lo que el gobernador Pedro Bravo de Acuña (1602-1606) culparía a la Audiencia del levantamiento y masacre de 1603, aludiendo a la flexibilidad en la concesión de licencias. . Las cifras sobre aquella inmigración, tanto chinas como españolas, son bastante coincidentes. A principios del siglo XVII se calculaban entre 20.000 y 30.000 sangleyes, ${ }^{10}$ originarios en su mayoría de Fujian, ${ }^{11}$ lo que las fuentes de la dinastía Ming explicaban que «por estar muy cerca y ser ricas las Filipinas, más de diez mil comerciantes de Fujian se fueron y muchos de ellos no volvieron, quedándose de forma permanente allí donde también tuvieron sus hijos». ${ }^{12}$

Aquel aumento de la población china acabó por generar desconfianza entre los hispanos, dando lugar a una política segregacionista cuya principal manifestación tuvo lugar en 1581, cuando Gonzalo Ronquillo mandó construir en el norte de Manila el primer Parián, con sus propias autoridades, aunque asumiendo las responsabilidades tributarias impuestas por las autoridades españolas. ${ }^{13}$ Estas, además, eran conscientes de que la actividad de los chinos era la principal fuente de riqueza del archipiélago, que, en contrapartida, abastecía de plata americana al imperio oriental. ${ }^{14}$

Otro aspecto que no debe obviarse es el origen de Fujian de los inmigrantes, especialmente desde 1567 , cuando tras una política de aislamiento del imperio, conocida como «Hai jin»o «Prohibición del mar»,

Los tomos que van del $100 .^{\circ}$ al $112 .^{\circ}$, tiene una relación detallada de los personajes importantes, tanto los familiares reales como los oficiales. Los tomos del $113 .^{\circ}$ al $332 .^{\circ}$ se utilizaron para dar cabida a las figuras menos importantes y los asuntos exteriores del imperio oriental. Cabe señalar que en el tomo $213 .^{\circ}$ se apuntaron las actividades de cuatro países europeos: Portugal, España, Italia y Holanda. Ming shi se halla íntegramente transcrita en «Zhong guo zhe xue shu dian zi hua ji hua (Plan de obras filosóficas electrónicas de China)», disponible en: http://ctext.org/wiki.pl?if=gb\&res=410835\&remap=gb [Consultado: 10/01/2021].

8 San Agustín, 1975 [1698], 329-330.

9 García-Abásolo, 2008, 359-360.

10 Huang y He, 2016, 99. García-Abásolo, 2012, 29.

11 Zhuang y Chen, 2012, 112-113.

12 Ming shi (Historia de Ming), vol. 211. En la bibliografía española también se confirmó que durante el siglo XVI casi todos los comerciantes chinos que acudían a las Filipinas eran de Fujian, véase Gil, 2011, 35 .

13 Sobre el Parián puede verse Alva Rodríguez, 1997, 55-72.

14 En cuanto a la bibliografía sobre este tema, además de las obras ya citadas, al redactar este trabajo, hemos tenido en cuenta a Boxer, 1969; Chaunu, 1960; Díaz-Trechuelo, 2001; Gayo Aragón, 1950; Gorriz Abella, 2011; Headley, 1995, entre otros. 
consecuencia de las peticiones reiteradas de la mencionada provincia costera y la de Cantón, se accedió a abrir el puerto de Haicheng como único autorizado para comerciar con el exterior. ${ }^{15}$ Sin embargo, debemos recordar que las prohibiciones previas no habían impedido a los de Fujian mantener un comercio de contrabando, pues como decía Xu Fuyuan en el siglo XVI, «sin la navegación y el comercio exterior le faltarán la ropa y la comida al pueblo. A la gente de Fujian le gusta vivir del mar, pese al peligro, porque así es la tradición en esta zona, sobre todo en la ciudad de Zhangzhou». ${ }^{16} \mathrm{Su}$ presencia masiva implicó unas influencias culturales en Filipinas que aún no han sido estudiadas en profundidad.

Otro fenómeno que debemos tener en cuenta es la política fiscal del imperio en unos momentos de grave crisis. Tradicionalmente los chinos pagaban sus impuestos en fuerza de trabajo o en especies, sobre todo cereales. Sin embargo, algunos cambios permitieron en determinados lugares que se pagaran en plata, lo que convirtió a este metal en una materia fundamental para la economía imperial; por eso, fray Gaspar da Cruz decía a mediados del siglo XVI, «[...] na China não há moeda de ouro nem de prata, senão que correa peso o ouro e a prata, e a peso se compra e vende tudo, tudo homem tem balanças e pesos em sua casa, o que tudo é mui perfeito em muita maneira». ${ }^{17}$ En 1573 aquel sistema contributivo se generalizó a todo el imperio, conociéndose como el «Yi tiao bian»o «Azote único». ${ }^{18}$ Esto supuso un avance para la economía, pues los campesinos pudieron optar por el cultivo de plantas industriales como algodón, morera y lino, que contribuyeron al desarrollo manufacturero y con ello el comercio exterior y la entrada de plata. Además, el estado se beneficiaba también con sus gravosos impuestos a la importación argentífera, cobrando $150 \ll$ 《liang $»^{19}$ a cada barco, tal y como nos relata Zhang Xie, un autor de la época. ${ }^{20}$ Aquellos cambios, además, hicieron que la plata americana llegase hasta el gran imperio oriental,

15 Haicheng fue el antiguo puerto Yuegang —en castellano Puerto de la Luna—. En 1548, la autoridad Ming amplió el territorio de Yuegang convirtiéndolo en Haicheng.

16 Xu, 1962, 4333. Xu Fuyuan (1535-1604) fue nombrado gobernador de la provincia Fujian en 1592.

17 Cruz, 1997 [1569-1570], 152.

18 En cuanto a los cambios y a las transformaciones de la dinastía Ming, además de las fuentes primarias, hemos consultado, entre otras, las obras de Brook, 2010; Spence, 2011; Fan, 2012; Li, 2018.

19 «iang» era la medida de peso que se usaba en la China antigua y se conocía en las fuentes occidentales como «tael». Un «liang» equivale a 37,3 gramos.

20 Zhang, 1981 [1617], 132. Zhang Xie (1574-1640), natural de Longxi de la ciudad Zhangzhou, fue uno de los escritores más importantes de la provincia de Fujian entre los siglos XVI y XVII. Vivió toda su vida en su tierra natal y nos legó unos escritos indispensables para investigar la historia de Fujian y de la dinastía Ming durante dicha época. 
siendo casi el único producto que los españoles pudieron aportar, como lo expresaba en el siglo XVII Gu Yanwu al decir que «los chinos que venden productos en Luzón, lo hacen solo a cambio de plata». ${ }^{21}$

\section{«Hay mucho oro allí en Luzón»: los miembros de la embajada en busca del oro}

Con el fin de negociar y de realizar intercambios con los comerciantes orientales, se exigió un quid pro quo. En las Filipinas los españoles no pudieron disponer de los recursos adecuados, ni en calidad ni en cantidad, como para atraer a los chinos. No obstante, paralelamente a la expansión española en las islas, en América la minería estaba en pleno auge, en buena medida gracias a la aplicación de la tecnología del proceso de amalgamación en frío, por lo que la producción de plata alcanzaba unos niveles sin precedentes. Así pues, aquel fue casi el único producto de interés para el comercio de los asiáticos, ${ }^{22}$ que intercambiaron con mercancías de lujo, provocando una masiva llegada de plata al puerto de Haicheng, convertido en uno de los lugares más importantes de abastecimiento de aquel mineral al imperio Ming.

En cuanto a la cantidad de la plata que se llevó en este periodo hasta China, a pesar de la dificultad que existe para calcularlo, algunos investigadores han aventurado algunas cantidades. André Gunder Frank, por su parte, dedujo que entre el siglo XVI y mediados del siglo XVII, la producción de plata en América debió estar en torno a las 30.000 toneladas, de las que entre 7.000 y 10.000 acabaron en China. ${ }^{23}$ Esta estimación ha sido confirmada por los investigadores chinos, utilizando sus propias fuentes. Según Wan Ming, uno de los especialistas más reconocidos en estas pesquisas, desde 1571 hasta 1644, en China habrían entrado un total de 7.620 toneladas de plata americana. ${ }^{24}$ Es más, los autores coetáneos de los Ming llegaron a constatar la utilización generalizada de la moneda española en

$21 \mathrm{Gu}, 1985,33 B$. Gu Yanwu (1613-1682) fue uno de los escritores más famosos durante los últimos años de la dinastía Ming y los primeros años de la dinastía siguiente, Qing.

22 Según los estudios realizados, en China se consumían maderas, nidos de pájaros, adornos de coral, oro, perlas y algodón, entre otros productos filipinos. Sin embargo, durante la dinastía Ming se contempló una nueva y creciente demanda de plata, teniendo en cuenta que este metal precioso llegó a ser en China el medio de pago más importante desde el siglo XV hasta los primeros años del siglo XX.

23 Frank, 2008, 181

24 Wan, 2004, 151. 
Fujian. Un autor de la época nos dice que «la moneda [española] se acuñó en plata y en su cara aparecían inscripciones en caracteres extranjeros [para los chinos]. La moneda contiene un 96 por ciento de plata y en la actualidad es muy usada por la población de Zhangzhou». ${ }^{25}$

A diferencia de la plata, el oro no era tan imprescindible para los negocios entre chinos y españoles. Por un lado, en el archipiélago apenas se podía encontrar este metal precioso, como lo reconocieron los oficiales reales en una misiva enviada a Felipe II en 1578: «Los soldados saquearon el pueblo, encontrando mantas y gallinas, pero muy poco oro, pues toda su riqueza es el comercio con Siam, Patan, China, Mindanao y Joló, y con los portugueses que vienen de Malaca». ${ }^{26}$ En ese sentido, el utilizar oro para el comercio hubiese resultado más provechoso, puesto que en siglo XVI la relación entre la plata y el oro en la China de los Ming era de cinco a seis por uno, y en el mercado europeo de catorce a uno. ${ }^{27}$ Sin embargo, el monarca español prohibió el uso del oro para contratar con los asiáticos, tal y como le había sido aconsejado: «[...] no se saque oro de aquellas yslas para la China, sino que en precio y paga de las mercaderías que compraron a los chinos les den tostones, cera, y algodón de lo qual ellos reciuieran más contento y vienen de mejor gana». ${ }^{28}$

La producción aurífera en las Filipinas era muy escasa, pues no sería hasta varias décadas después cuando se descubriesen algunos yacimientos de cierta importancia, como «oro de superior quilate» o «exquisito polvo».$^{29} \mathrm{El}$ poco que se obtenía por entonces estaba prohibido exportarlo hacia el Celeste Imperio, donde las fantasías de algunos chinos habían despertado la idea de un Dorado oriental. Este iba a ser el motivo que incitó a un sangley a plantear su control. Así, Zhang Yi logró que su propuesta llegara a oídos del emperador Wanli, ${ }^{30}$ quien tuvo el reinado más prolongado de los Ming y en cuyo tiempo se iniciaron los contactos con los castellanos. Aquel emperador había adoptado una forma de gobernar muy peculiar, pues en el periodo que va de 1589 a 1615 no convocó ni una sola audiencia general.

$25 \mathrm{Gu}, 1985$, 400A.

26 «Carta de los oficiales reales sobre varios asuntos, Manila, 28 de julio de 1578», Archivo General de Indias, Sevilla (AGI), Filipinas, 29, n. 29.

27 Bouza, 1987, II: 627.

28 «Comercio con China y situación de las Filipinas, Logrosán, 24 de abril de 1580», AGI, Filipinas, 339, 1. 1, ff. 187r-190v.

29 Malo de Luque, 1790, 329.

30 El nombre original de este emperador fue Zhu Yijun y el nombre póstumo que se le dio fue el de Shenzong. 
Aparte de los temas relacionados con la cuestión de impuestos y de defensa, apenas respondía a las propuestas de sus ministros y acostumbraba a dejar sin resolver la mayoría de los asuntos imperiales, de modo que durante los últimos treinta años de su reinado tan solo mantuvo cinco entrevistas personales con los altos dignatarios. ${ }^{31}$ Era la venganza para bloquear e ignorar la administración tras los ataques y contrataques de los moralistas después de la muerte de su secretario Zhang Juzheng. ${ }^{32}$

En los años noventa del siglo XVI, algunos factores externos e internos alteraron la vida imperial. Fueron especialmente decisivas las llamadas «Tres guerras de Wanli»,33 así como el incendio que destruyó los dos palacios imperiales, del emperador y de la emperatriz, en 1596. A ello se añadía que era necesario mantener un ejército formado esencialmente por mercenarios, lo mismo que a un gran número de príncipes. ${ }^{34}$ Aquellos sucesos dieron lugar a dificultades financieras, difíciles de afrontar con la dejadez gubernamental de Wanli, al que tuvieron que obligar a tomar medidas extraordinarias. Según la fuente imprescindible para investigar la dinastía Ming, Ming shi lu,,$^{35}$ el emperador llegó a confesar que «hoy en día el país está en un apuro, se agota la riqueza tanto pública como privada». ${ }^{36}$ Para recuperarse de esta situación, el 14 de julio de 1596 un oficial militar de Pekín, propuso «explotar las minas para conseguir el dinero suficiente para la gran obra $»^{37}$ que suponía la reconstrucción de los dos palacios. Mientras

31 Schirokauer y Brown, 2006, 255. Huang, 2007, 221-235.

32 Fairbank, 1997, 178.

33 En los años noventa del siglo XVI, la dinastía Ming se vio obligado a emprender una serie de actividades militares contra los diferentes enemigos de las distintas zonas, las cuales han sido denominadas por los historiadores como las «Tres guerras de Wanli»: La guerra de Bozhou contra la revuelta de los aborígenes liderados por Yang Yinglong en el suroeste de China entre 1590 y 1600; la guerra contra los mongoles en E'erduosi en 1592; y el envío de los soldados chinos para participar en la guerra contra los japoneses en el territorio de Corea entre 1592 y 1598 . Véase Mote y Twitchett, 1992, 544-554.

34 Escalante, 1991 [1577], 65-66. Botton Beja, 2008, 321.

35 Ming shi lu en español Verdaderos registros de Ming. Se trata de los registros elaborados por los cronistas cortesanos de la dinastía Ming, anotando fielmente por orden cronológico no solo los edictos, instrucciones y leyes, sino también los acontecimientos importantes de cada reinado. Consta de 13 libros y 2911 volúmenes, organizados por el orden cronológico de los emperadores. Entre ellos se hallan 596 volúmenes dedicados a los registros del reinado de Wanli bajo el subtítulo de Ming shen zong shi lu (Verdaderos registros de Ming shen zong). Esta obra histórica de unos dieciséis millones caracteres fue elaborada por el colectivo de cronistas oficiales asignados por los diferentes emperadores. Se halla Ming shen zong shi lu íntegramente transcrita en «Zhong guo zhe xue shu dian zi hua ji hua (Plan de obras filosóficas electrónicas de China)», disponible en: https://ctext.org/wiki.pl?if= gb\&res $=964038 \&$ remap $=$ gb [Consultado: 10/01/2021].

36 Ming shen zong shi lu (Verdaderos registros de Ming shen zong), vol. 298.

37 Idem. 
tanto el emperador recurrió al envío de un gran número de eunucos ${ }^{38} \operatorname{como}^{2}$ recaudadores de impuestos y a la vez investigar sobre depósitos minerales $\mathrm{y}$ otras fuentes de riqueza. ${ }^{39}$

Así pues, a finales del siglo XVI y en los primeros años del siglo siguiente, en China se generó un interés colectivo por la riqueza, que afectó desde el propio emperador hasta el pueblo llano. Fue en esas circunstancias cuando surgió la figura de un comerciante, natural de la isla de Wuzhou, que se hallaba en el distrito de Haicheng, formando parte de la ciudad de Zhangzhou, en Fujian. Este, aprovechando aquellas circunstancias se presentó en Pekín con una propuesta para tratar de explotar un supuesto Dorado, que no era sino una montaña de oro existente en las Filipinas. Él había vivido en las islas como sangley, ejerciendo el oficio de carpintero, «aviéndose hecho hombre de bien». ${ }^{40}$ Esta experiencia personal y la entrada de gran cantidad de la plata española en China hicieron, sin duda alguna, que las proposiciones que hizo fuesen mucho más creíbles.

Habiendo atravesado una larga distancia de más de dos mil kilómetros, en julio de 1602 Zhang Yi llegó a Pekín. De acuerdo con las costumbres imperiales de la dinastía Ming, junto con un oficial militar de baja graduación en la capital, Yan Yinglong, entregó una propuesta dirigida al emperador, en la que se decía que:

fuera de la provincia de Fujian hay una isla que se llama Luzón. Allí se encuentra la montaña Jiyi [Cavite], en donde se deposita una gran cantidad del oro y la plata. Si su Majestad manda a los suyos que la exploten, cada año pueden llevar 100 mil liang de oro y 300 mil liang de plata. En la montaña de Jiyi hay también un árbol muy especial que tiene en las ramas hojas del oro. ${ }^{41}$

Como podemos apreciar, el mito occidental del árbol de las hojas de oro que aparece en la Eneida de Virgilio, ${ }^{42}$ así como el de la montaña de oro, también tenían su referente en el mundo sínico, como lo prueba la

38 Los eunucos, desde los primeros emperadores de la dinastía Ming, iban acumulando cada vez más poderes. En los años en los que gobernaba Wanli, debido a su mala relación con los burócratas, los eunucos fueron asignados a algunas tareas que deberían asumir los ministros, convirtiéndose en jefes de almacenes en el palacio, supervisores de talleres imperiales, administradores de los tributos, etc. En el siglo XVII, el número total de este grupo alcanzó hasta 10.000 trabajando en la capital y 70.000 en las provincias. Véase Botton Beja, 2008, 300-301; Buckley Ebrey, 2009, 249.

39 En cuanto a la situación económica y las nuevas medidas fiscales de Wanli en los años noventa del siglo XVI, véase Fang, 2012, 108-113; Gernet, 1999, 384-386.

40 Citado en Gil, 2011, 468.

41 La propuesta fue recogida en Zhang, 1981 [1617], 255.

42 Virgilio, 1985 [s. I a. C.], 153. 
proposición que se hizo al emperador Wanli. Frente a aquella credulidad, un funcionario llamado Wen Chunya, que actuaba como «duyushi», vigilando la integridad de la burocracia en la Corte, al enterarse de la mencionada propuesta la rebatió con firmeza:

\begin{abstract}
Yan Yinglong y Zhang Yi han informado que hay en el ultramar 100 mil [liang] $]^{43}$ del oro y 300 mil [liang] de la plata, lo que no deja de ser absurdo, pero, aún siendo su Majestad muy sabio, también lo ha creído. Nosotros, los ministros estamos tan sorprendidos que apenas podemos dormir ni comer, pues esto será una causa para que se inicie una guerra [con los extranjeros] y gran parte del tesoro imperial se consumirá inútilmente. Si no podemos ganar la guerra, además de la riqueza, se perderá aún más [...] Aunque la montaña Jiyi está en el mar, no es posible que esté allí llena del oro y la plata que todo el mundo pueda recoger libremente [...] Yan Yinglong y Zhang Zhi deberían ser castigados. ${ }^{44}$
\end{abstract}

A pesar de que el emperador Wanli se mantenía indiferente a la mayor parte de los temas administrativos, ya que, como mencionamos, muchos quedaron sin resolver durante los años de su largo reinado, una propuesta de este calibre resultaba tentadora, pues podía ser una solución para los apuros económicos a los que se estaba enfrentando el imperio. En consecuencia, después de leer la sugerencia de Zhang Yi, reaccionó de inmediato y ordenó que los oficiales locales de Fujian organizaran una embajada para investigar el asunto de las minas de Luzón. En ello debió influir también su afición a la alquimia, por lo que se vería tentado por la hipotética montaña de oro. Recordemos en tal sentido, que eran los tiempos en que Mateo Ricci y Diego de Pantoja - a los que se atribuían conocimientos sobre aquella protociencia - se hallaban en China, donde eran muchos los que, como su emperador, sentían debilidad por aquellos saberes: «entre los chinos se hallan muchos tan dados al arte de la alquimia, que casi pierden su juizio en descubrirla i apurarla, porque se persuaden que el azogue puede convertirse en verdadera plata por virtud de cierta ierba». ${ }^{45}$

A través de las Crónicas locales podemos apreciar y rastrear el proceso y la evolución de la propuesta de la búsqueda del oro. Los datos nos aparecen tanto en las del gobierno provincial de Fujian como en las de otras

43 En los documentos originales no se dan medidas concretas, pues con la información de 100, 000 del oro y 300, 000 de la plata suponemos que se está refiriendo a «liang». De todos modos, el autor querría expresar que en las Filipinas existía una gran cantidad de metales preciosos.

44 Ming shen zong shi lu (Verdaderos registros de Ming shen zong), vol. 374

45 Trigault, 1621, 97. 
ciudades, sobre todo Zhangzhou, y las de los distritos, tales como Haicheng de donde salían las naos chinas con destino a las Filipinas:

Yi frecuentaba [las islas Filipinas] y sabía que allí estaban asentados aproximadamente unos diez mil comerciantes chinos. En consecuencia quería trasladarse allá con las instrucciones regias y, con la excusa de explotar las minas, intentaba un saqueo de la isla. Al recibir la orden del emperador, los funcionarios de Fujian se sintieron muy sorprendidos, pero finalmente decidieron enviar al gobernador de Haicheng que acompañase a la embajada y que hiciese investigaciones sobre aquel asunto. ${ }^{46}$

Las fuentes indican que, desde un principio, pese a la decisión firme de Wanli, los oficiales de Fujian estaban en desacuerdo con la proposición de Zhang Yi. Sea como fuere, las autoridades de aquella provincia de Fujian no podían resistirse a la resolución del emperador, que «envió al "xiancheng" de Haicheng, Wang Shihe, y a Yu Yicheng que detentaba el cargo de "baihu"», ${ }^{47}$ considerando que eran los oficiales que tenían los mayores conocimientos sobre las Filipinas. Como se trataba de una embajada para informarse de unas supuestas minas con las que conseguir nuevos ingresos para la Corte, no podía estar ausente el eunuco Gao Cai, encargado por Wanli de gestionar los asuntos tributarios y de metales preciosos en Fujian, por lo que según Ming shi lu, siguiendo la instrucción del emperador, también participó en la misión de investigar del oro filipino..8

Múltiples fuentes de la dinastía Ming nos ponen de manifiesto que los oficiales chinos, los consejeros en Pekín y las autoridades locales de Fujian se habían opuesto taxativamente a las intenciones de Zhang Yi; pero el emperador insistió en que se mandara una delegación para investigar el asunto y aquella embajada no tuvo otra opción que la de trasladarse a Manila. Por tanto, la propuesta de Zhang Yi había sido finalmente aprobada por Wanli, aunque, como no consiguió los navíos que solicitó al rey para cargar el oro, el exsangley tuvo que acompañar en la misión a los mencionados mandarines y al eunuco, que le llevaron encadenado. ${ }^{49}$ "Y si así no fuese [haber oro] lo castigase con la vida, que venían a llevar a su rey averiguación de lo que en aquello había». ${ }^{50}$

46 Chen, 1715, vol. 34.

47 Cai y Zhang, 1762, vol. 21. «Xiancheng» era vice-gobernador del distrito Haicheng, pues al mismo tiempo «baihu» era un cargo militar local, equivalente al centurión.

48 Ming shen zong shi lu (Verdaderos registros de Ming shen zong), vol. 390.

49 Morga, 2007 [1609], 176.

50 Idem. 
La embajada, por tanto, la componían además de los instigadores del proyecto Zhang Yi y Yan Yinglong, un oficial gubernamental, un militar y un eunuco; es decir, representantes de los tres grandes poderes de los últimos años de la dinastía Ming. Todos ellos emprendieron el viaje rumbo a aquellas islas cercanas, en donde vivían hacía varios años aquellos chinos a los que se denominaba sangleyes.

\section{La presencia de la embajada en las islas}

Habiendo recuperado los nombres y datos personales de los embajadores enviados desde China para buscar el oro en Luzón, las fuentes elaboradas en las Filipinas en los primeros años del siglo XVII ${ }^{51}$ nos resultan de gran utilidad para saber qué es lo que se encontró la legación en el archipiélago, pues la visita repentina e inesperada de aquellos mandarines iba a tener graves repercusiones e incluso provocó grandes polémicas.

Las naves procedentes de los puertos de Fujian solían llegar a la bahía de Manila durante el mes de marzo, en la época favorable del monzón. Sin embargo, en 1603, los chinos no aparecieron en las costas filipinas hasta los últimos días de mayo, según comunicó al rey Felipe III el gobernador de las islas, Pedro de Acuña, el 3 de junio del mismo año: «al fin llegaron catorce nauios con mercadurías y en uno dellos tres mandarines, que son como jueces o comisarios, capitanes o personas que tienen gouierno y mando, y desde la mar me enuiaron una carta en su lengua que traducida en la nuestra». ${ }^{2}$

El retraso, por tanto, era probable que tuviera algo que ver con la mencionada embajada, cuya preparación exigiría más tiempo. La señalada misiva fue firmada por Chancian - Yu Yicheng en chino-, el oficial militar de Haicheng. En cuanto a la datación de la carta, en la traducción española se registró como «en 31 años del Reyno de Vandec a los diez de la quarta luna que es la presente de mayo según se quenta», ${ }^{53}$ lo que se corresponde

51 En cuanto a la embajada china, tenemos tres obras escritas en el siglo XVII en las Filipinas por Francisco Colín, Antonio de Morga y Bartolomé Leonardo de Argensola respetivamente. Los tres autores nos legaron unas descripciones detalladas y confiables. También nos sirven como referencia algunos estudios. Véase Borao, 1998a, 25-28; Gil, 2011, 468-471.

52 Colín, 1900 [1663], 413.

53 Ibidem, 414. La fecha se registró según el calendario chino, pues «Vandec» sin duda alguna se refiere al rey chino Wanli. 
con el 2 de mayo de 1603 del calendario juliano, fecha muy anterior a su llegada a Manila. ${ }^{54}$

Los representantes de Wanli intentaban explicar al gobernador que su presencia tenía únicamente que ver con la existencia de oro en una isla llamada en las fuentes en español Jiyi, Cavite o Keit y que «no ay otro negoçio más que este y así podrá el governador de Luzón dormir siguro y sin reçelo y no sospechar mal». ${ }^{55}$ Además, habían alegado que aquella montaña no tenía dueño por estar en medio del mar. ${ }^{56}$

La carta original y la traducida al español llegaron a las Filipinas cuatro días antes que su autor, por lo que el gobernador y los habitantes del archipiélago se enteraron de que, además de los sangleyes, ese año llegarían unos mandarines. El 23 de mayo de 1603, junto con los barcos comerciales, una gran nave en la que viajaban los miembros de dicha embajada apareció frente a Manila. En un primer momento, aquellos oficiales chinos fueron bien recibidos, a pesar de las dudas e inquietudes que había causado su presencia. «El gobernador les dio licencia para que saliesen del navío y entrasen en la ciudad con su acompañamiento». ${ }^{57}$ Es más, según la fuente oficial de Ming, para tratar a los chinos como enviados del emperador, el gobernador no solo mandó que «se tapizase de flores su camino sino que también les ofreció una nutrida escolta de soldados», ${ }^{58}$ probablemente más por controlar sus movimientos que por deferencia diplomática.

Al hablar de la finalidad real de la embajada, hay que hacer un balance cuidadoso de acuerdo con el emperador y los diferentes protagonistas que la integraban, pues cada uno de ellos tenía fines e intereses distintos. En cuanto a Wanli, la información de la probable existencia de aquel oro le había deslumbrado justo en el momento en que su dinastía estaba en un grave apuro financiero; por tanto, la producción aurífera le podría servir para solucionar sus dificultades económicas, tal y como lo expusieron los embajadores en la carta destinada al gobernador Pedro de Acuña: «[...] cada año podía llevar al rey de China çient mill taes de oro, y de plata treçientos mill taes para su gasto, para que sus vasallos no pagasen tributo ni fuesen molestados» ${ }^{59}$ El problema en todo esto estaba en que la supuesta explotación

54 «Carta de mandarines de China a Acuña sobre el oro de Cavite, desde el mar, 10 de mayo de 1603», AGI, Filipinas, 7, r. 1, n. 6.

55 Idem.

56 Idem.

57 Morga, 2007 [1609], 174

58 Ming shi (Historia de Ming), vol. 211. Gil, 2011, 469.

59 Colín, 1900 [1663], 414. 
debía hacerse en un espacio que pertenecía a la Corona de Castilla y, aunque aquella fantástica montaña estuviese en el mar, las autoridades españolas eran contrarias a la teoría del mare liberum que expondría unos años más tarde Hugo Grocio. ${ }^{60}$

En cuanto al promotor del proyecto, Zhang Yi, no parece que hubiese motivos aparentes para informar a su monarca de noticias exageradas e incluso falsas. Sin embargo, sus contemporáneos, tanto chinos como españoles, se dieron cuenta de que sus intenciones verdaderas estaban en relación con sus intereses personales. Así, un oficial e intelectual local de Haicheng, Gao Kezheng, denunció las consecuencias de las pretensiones de Zhang Yi en un escrito que se incluiría en la crónica de dicho distrito: «Para recoger el oro en el mar, se ve obligado a enviar unos diez barcos y a reclutar a más de mil trabajadores. ¿Quién puede preparar y hacerse responsable de los barcos y las personas? [...] Para poner en práctica este plan, satisfaciendo las expectativas del rey, tiene que expropiar las naves comerciales, en detrimento de la riqueza de los pueblos». ${ }^{61}$

También en Manila había quien tenía conocimiento tanto del personaje como de su verdadero propósito; así, el 5 de julio de 1603, dos días después de la llegada de los chinos, el arzobispo Miguel de Benavides escribió avisando a Felipe III: «Un chino de los que aquí vienen, que es sillero y carpintero, volvió a China [...] fingieron y dixeron mil mentiras arrimadas a una verdad, todo con ánimo y deseo que el rey de China les diese licencia para juntar gente para salir a la mar y una vez puestos en ella venir a conquistar esta tierra, o hazerse piratas y robar en la misma China o donde pudieran». ${ }^{62}$ Las finalidades explicadas en la carta y comunicadas por los miembros de la embajada durante la ceremonia de bienvenida, no solo no lograron persuadir a las autoridades hispanas, sino que la presencia de los mandarines en el centro neurálgico del poder en el archipiélago, que era Manila, suscitó profundas sospechas y levantó alarmas entre los españoles de toda condición. La historia oficial de la dinastía Ming contiene unas pinceladas sobre la mencionada entrevista entre los españoles y los visitantes inesperados desde el gigante vecino en el norte:

El mandatario de Luzón preparó un banquete y preguntó a Shihe [el mandarín chino] y a otras personas que llegaron, si el Celeste Imperio iba a enviar a su gente para

60 Grocio, 1609.

61 Cai y Zhang, 1762, vol. 21.

62 Colín, 1900 [1663], 415. 
explotar las minas de aquel lugar. Pero esas montañas tienen sus dueños, ¿cómo pueden ustedes explotarlas? Y si en China hubiese tales montañas, ¿nos sería permitido explotarlas?

Y dijo lo de que en los árboles hay hojas de oro, ¿pero de qué árboles se trata?

Shihe no pudo responder y miró varias veces a [Zhang] Yi.

Yi contestó: Esto está lleno de oro, ¿por qué pregunta de dónde vienen las hojas?

Todos los que estaban presentes se echaron a reír, momento en el que los españoles iban a detener a Yi para matarlo. ${ }^{63}$

Frente a los chinos, la opinión de Bartolomé Leonardo de Argensola es muy representativa de las ideas generalizadas entre sus coterráneos asentados por entonces en las Filipinas: «Claro está que la venida destos mandarines auía de parecer sospechosa, y que trayan intento diferente del que publicaron. Son los chinos agudos y rezelosos, y no era creyble que su rey los embiasse a lo que dezían, ni verisimilla ficcion para darla a entender a los españoles». ${ }^{64}$

Esas dudas las expresó igualmente Bernardino del Castillo Maldonado, castellano de la fortaleza de Santiago, en Manila, exponiendo que lo que pretendían aquellos embajadores era facilitar la toma de Filipinas con una armada, por lo que previamente deseaban comprobar las defensas de $\mathrm{Ca}$ vite, siendo conveniente, por tanto, fortificar aquel puerto. ${ }^{65}$ No cabe duda que unos 20.000 sangleyes y la presencia de mandarines hacían saltar las alarmas, que con posterioridad dieron lugar a revueltas, represión y expulsiones ${ }^{66}{ }^{6}$ Sin embargo, en las fuentes chinas nada parece indicar que hubiese una intencionalidad perversa y que se hable de un «ridículo pretexto». ${ }^{67}$ Lo que parece claro es que la diplomacia imperial no era tan burda como para actuar de aquella manera si hubiese otras intenciones. No podían decir lo mismo los hispanos entre los que no faltaban planes de invasión desde las Filipinas ${ }^{68}$ Así lo había pensado el gobernador Francisco de Sande ${ }^{69}$ y lo mismo mantenía, aunque con ideas cristianizadoras, el visionario jesuita Alonso Sánchez, que abogaba por la implantación de la fe por la fuerza, ${ }^{70}$

63 Ming shi (Historia de Ming), vol. 211.

64 Argensola, 1609, 316.

65 «Carta de Bernardino del Castillo sobre llegada de tres mandarines, Manila, 7 de julio de 1603», AGI, Filipinas, 35, n. 62.

66 Esto puede verse en García-Abásolo, 2011, 223-242.

67 Jordana y Morera, 1888, 10.

68 Cervera Jiménez, 2013, 207-234. Ollé Rodríguez, 2002. Vega y de Luque, 1979; 1980; $1981 ; 1982$.

69 Rojas, 2019, 41.

70 Por sus posiciones, se le ha considerado como el primer ideólogo que plantea la cristianización de China por medio de la conquista. López-Gay, 2005, 128. 
frente a posiciones como la del humanista Pedro de Valencia, que criticaba aquella postura. ${ }^{71}$ Cierto es que aquellas pretensiones tenían sus antecedentes, por ejemplo, en Martín de Rada, que según algún autor elaboraría el primer proyecto concreto de conquista. ${ }^{72}$

Además de la finalidad que les resultaba difícil de creer, a los españoles también les pareció pintoresca y curiosa la presencia de los mandarines chinos, sobre todo por la forma en la que vestían y se movían por la ciudad durante su estancia. Era la oportunidad que tenían los colonizadores de contemplar de cerca algunos rituales en el comportamiento de los miembros de las élites de una cultura milenaria y esplendorosa, de la que solo se tenía conocimientos a través de algunas obras y de los viajeros, especialmente los portugueses. Con aquella presencia muchos europeos se situaban por primera vez frente a lo real y maravilloso del mítico imperio.

Cabe señalar que la primera imagen que se tuvo de los visitantes, ya al salir del navío, fue la manera en la que eran tratados por sus súbditos. «Desembarcados, con largo acompañamiento de los suyos, en sillas de marfil y de otras maderas tersas y doradas, sobre los ombros de sus criados, que andauan vestidos de colorado, fueron derechos a las casas reales». ${ }^{73}$ Los mandarines no bajaron de las sillas hasta las puertas del que era su destino, la sede de la Real Audiencia de las Filipinas, en donde el gobernador Pedro de Acuña, junto con los capitanes y soldados, estaban esperándoles para tener una primera audiencia con ayuda de los intérpretes chinos: «Los apearon de las sillas y entraron a pie, dejando sus banderas, upos, lanzas y otras insignias de mucha demostración que traían, en la calle, hasta una sala grande bien aderezada, donde el gobernador los recibió en pie, haciéndole muchas humillaciones y cortesías los mandarines a su usanza». ${ }^{74}$ Esa era la imagen que daba un funcionario español. ¿Pero qué podían pensar aquellos hombres de un espacio que en la época no gozaba de grandes lujos, al menos no comparables con la opulencia oriental? Y menos si lo relacionamos con las riquezas de los palacios de los mandatarios, en los que se ponían de manifiesto los tesoros y expresiones artísticas del imperio Dorado, nombre con el que se conocía al de la dinastía Ming.

Se alojaron finalmente en unas casas que les fueron asignadas dentro de Manila por el gobernador. Una vez que estuvieron asentados en la

71 Viforcos Marinas, 2012, 367-573.

72 Ollé Rodríguez, 2002, 41

73 Argensola, 1609, 315.

74 Morga, 2007 [1609], 174. 
ciudad, sus movimientos fueron vigilados muy de cerca por los españoles y el fiscal de la Audiencia, Jerónimo de Salazar, describió detalladamente la manera en la que se movían por las calles de Manila:

salen por esta ciudad de sus casas sentados en unas sillas en hombros de quatro sangleyes, e junto a sus personas de una vanda, e de otra traen seis de su guardia con armas como archeros, e delante de si lleuan dos sangleyes que de los hombros lleuan colgada una caxuela en que dicen van las chapas de ser tales mandarines que es lo que acá llamamos prouisiones y títulos reales. Detrás va otro sangleye a caballo que dize es el escribano de los mandarines y delante dellos van en ordenanza seis sangleyes con unos bastones al hombro y en la cabeza dellos unas tablillas blancas con unos caracteres de oro que dizen es insignia de alguaciles. Y otros seis sangleyes [llevan] unas vanderas pequeñas de diferentes colores y en ellas escriptos unos caracteres en lengua china que dizen demuestra grande autoridad de los dichos mandarines y su mucha jurisdicción. Y un sangley va con una caña gorda como el brazo barnizada de negro que dizen es ministro de justicia. E delante de la gente dicha van seis sangleyes que los dos lleuan dos cadenas de hierro que dizen es para echar a los que mandan prender, y los otros dos lleuan dos cordeles atados en unos bastones al hombro que dizen es para atar a los que mandan açotar, y los otros dos que llaman opos que es lo propio que aca verdugos que lleuan dos medias cañas de quatro dedos de ancho y una braça de largo con que açotan a los delinquentes que si quieren a pocos açotes los mata. ${ }^{75}$

En la comitiva de los mandarines, además de los subordinados que se encargaban de diferentes tareas y los múltiples objetos que se transportaban para demostrar su autoridad e identidad, también había un grupo de músicos que solían andar tocando un atabal y otros instrumentos orquestales. Junto con los músicos iban también dos pregoneros para evitar la aglomeración de personas: «otros dos sangleyes van entre estos que cada uno a veces va dando gritos en su lengua con un grande alarido, que dize va diziendo que se aparten, que vienen los mandarines». ${ }^{76}$

A pesar de las suspicacias desencadenadas por la presencia de aquellos visitantes en Manila, el gobernador, capitán general y presidente de la Audiencia, Pedro de Acuña, permitió que los enviados chinos entraran y se quedaran en la capital. No obstante, los oficiales de la Audiencia eran los menos pacientes con aquella presencia, protestando ante la máxima autoridad principalmente por dos cuestiones. Por un lado, porque las insignias que portaban los mandarines les inquietaban, lo que hizo que en dos ocasiones el fiscal Jerónimo de Salazar y el secretario Pedro Hurtado de Esquivel propusieran al gobernador la prohibición del uso de los símbolos de justicia

75 Colín, 1900 [1609], 418.

76 Idem. 
procedentes de otros reinos. Por otro lado, los chinos, pese a su corta estancia en Manila, fueron denunciados por ejercer la justicia inapropiadamente en los dos días que permanecieron: «El domingo en la tarde delante de la casa de uno de los dichos mandarines açotaron a un indio o mulato en la calle delante de la casa del dicho mandarín, estando él a la ventana todo en forma de justicia a usanza de China, y ayer lunes açotaron a un sangley en la propia manera y a otro dieron tormento en las manos todo a usanza». ${ }^{77}$

El gobernador se vio obligado a responder promulgando un auto que restringiera a los representantes imperiales el ejercicio de la administración de justicia, así como andar por la ciudad con las señaladas insignias, puesto que «destas solo le tienen y jurisdicción sobre todas las personas que residen en este archipiélago su señoría y las justicias del rey nuestro señor Don Felipe tercero, que es dueño y señor natural deste reyno». ${ }^{78}$ Con el fin de divulgar mejor las nuevas instrucciones, la orden se tradujo al chino y se colocó en el Parián, donde residían los sangleyes de Manila.

Los mandarines también eran conscientes de lo delicado de su presencia en aquella ciudad. Así, a los pocos días de la llegada, la embajada china solicitó una nueva audiencia con el gobernador, reiterando sus intenciones originales. «Los mandarines dijeron que bien entendían lo que el gobernador les trataba, pero que su rey les había mandado venir y les era fuerza obedecerle y llevarle respuesta, y que con hacer su diligencia habían cumplido, y se volverían». ${ }^{79}$

Finalmente, el gobernador Pedro de Acuña les facilitó el viaje a la montaña Jiyi, en la isla Cavite, que se encuentra a dos leguas de Manila. En presencia de los capitanes, los intérpretes y otros españoles, los mandarines interrogaron en público a Zhang Yi dónde se hallaba el oro: «El hombre respondió una palabra de coraçón harto brioso y díxoles si queréis que esto sea oro seralo, pero si queréis que no lo sea no lo será; lo que yo digo es que cortéis las cabeças a los yndios de esta tierra, y todo el cuello lo hallareis lleno de cadenillas y gargantillas de oro, y este es el oro que yo digo». ${ }^{80}$

$\mathrm{Al}$ escuchar las respuestas del chino, los españoles se irritaron tanto que quisieron darle muerte allí mismo, pero los mandarines consiguieron salvar la vida del farsante. Estos, ante la imposibilidad de regresar con noticias y muestras de aquel oro, ordenaron tomar una espuerta hecha de hojas

77 Ibidem, 420

78 Idem.

79 Morga, 2007 [1609], 176

80 Colín, 1900 [1663], 415. 
de palma de la tierra como prueba de haber pisado aquel lugar, en el que supuestamente deberían encontrarse muchos metales preciosos. Después de comer y descansar en la isla de Cavite, los chinos regresaron ese mismo día a Manila.

Pasados veinte días desde su llegada, todos los miembros de la embajada china se despidieron de los españoles, llevando la plata y los regalos del gobernador. Pocos días después de regresar, el vicegobernador de Haicheng, Wang Shihe, murió de la enfermedad que le había producido un ataque de pánico durante el viaje a las Filipinas. En cuanto a la persona que había dado lugar a aquella expedición, Zhang Yi, los mandarines le consideraron culpable del despropósito ante la Corte. En Ming shi y Ming shi lu, las dos historias oficiales de la dinastía Ming, se narra que fue decapitado en Pekín por orden del emperador Wanli, disponiendo que su cabeza colgase durante mucho tiempo en la costa de su tierra natal de Fujian para advertir a otras personas de intenciones malignas. ${ }^{81}$

\section{Conclusiones}

La embajada de los mandarines en 1603 coincide con el momento de revalorización de las islas Filipinas, toda vez que se comprobó la imposibilidad de conquistar el imperio oriental por las armas, especialmente en unos momentos en que la decadencia marítima de España era evidente y la casi imposibilidad de trasladar un contingente de ejército suficiente como para hacer frente al poderoso aparato defensivo de los Ming. En tal sentido, podemos recordar que, en 1598, solo para ayudar a Corea en su guerra con Japón, había movilizado cien mil soldados. Filipinas, de este modo, se había convertido en la única gran avanzada española en Oriente, necesaria también para el imperio de Wanli, como espacio para garantizar el abastecimiento de plata americana, del que había una gran necesidad tras los nuevos sistemas impositivos imperiales.

En ese ambiente, los mitos también surgieron en las Filipinas, ahora por parte de algunos chinos que, de alguna forma, repetían el esquema que se había producido con los españoles en las Indias. Es decir, el sueño de un

81 Ming shi (Historia de Ming), vol. 211. Ming shen zong shi lu (Verdaderos registros de Ming shen zong), vol. 404. Un informe enviado al gobernado de las Filipinas añadía que la cabeza había sido puesta dentro de una jaula. Véase Carta del visitador de Chincheo a Acuña sobre los chinos, Bandel, 12 de marzo de 1605, AGI, Filipinas, 7, r. 1, n. 21. 
Dorado que, para algunos crédulos, se ubicaba en algún lugar del mar en el archipiélago, pero cuya existencia no era admitida por el racionalismo de otros, especialmente de los funcionarios imperiales, poco proclives a las fantasías populares y que temían los peligros que podía generar aquel sueño, apoyado inicialmente por su propio emperador. Lo cierto es que la comprobación de la realidad mítica se llevó a cabo y se buscó la montaña de oro de Jiyi, que podía haber sido el sueño cumplido para muchos sangleyes y la solución a los problemas económicos de la dinastía Ming.

$\mathrm{Al}$ contrario de lo esperado y como ya sospecharon los mandarines, tal montaña de oro no existía, pero generó los recelos y miedos entre los españoles, numéricamente muy escasos, ante una supuesta invasión de las Filipinas. La consecuencia de todo aquello fue terrorífica, pues las suspicacias mutuas entre los chinos y los españoles dieron lugar primero a una rebelión organizada de los sangleyes. Para sofocarla, se produciría aquel mismo año de 1603 la primera masacre general de los inmigrantes chinos en Manila, en la cual se acabó con la vida de unas 20.000 personas.

Recibido, 23 de febrero de 2021 Segunda versión, 29 de abril de 2021 Aceptado, 9 de junio de 2021

\section{Referencias bibliográficas}

Alva Rodríguez, Inmaculada, Vida municipal en Manila, Córdoba, Universidad de Córdoba, 1997.

Argensola, Bartolomé Leonardo de, Conquista de las Islas Molucas, Madrid, Alonso Martín, 1609.

Borao, José Eugenio, «The massacre of 1603: Chinese perception of the Spaniards in the Philippines», Itinerario, 23:1, Leiden, 1998a, 22-39.

Borao, José Eugenio, «Percepciones chinas sobre los españoles de Filipinas: la masacre de 1603», Revista Española del Pacífico, 8, Córdoba, 1998b, 233-254.

Botton Beja, Flora, China: Su historia y cultura hasta 1800, México D. F., El Colegio de México, 2008.

Bouza Âlvarez, Fernando Jesús, Portugal en la monarquía hispánica (1580-1640). Felipe II, las Cortes de Tomar y la génesis del Portugal católico, Madrid, Universidad Complutense de Madrid, 1987.

Boxer, Charles Ralph, «Portuguese and Spanish Projects for the Conquest of Southeast Asia, 1580-1600», Journal of Asian History, 3:2, Bloomington, 1969, 118-136. 
Brook, Timothy, The Trouble Empire: China in the Yuan and Ming Dynasties, Cambridge, Harvard University Press, 2010.

Buckley Ebrey, Patricia, Historia de China, Madrid, La Esfera de los Libros, 2009.

Cai, Guozhen y Zhang, Xie, Hai cheng fu zhi [Crónica local de Haicheng], s.l., s.i., 1762.

Cervera Jiménez, José Antonio, «Los planes españoles para conquistar China a través de Nueva España y Centroamérica en el siglo XVI», Cuadernos Intercambio sobre Centroamérica y el Caribe, 12, San José, 2013, 207-234.

Chaunu, Pierre, Les Philippines et le Pacifique des Ibériques (XVI, XVII, XVIII siècles), Paris, S. E. V. P. E. N., 1960.

Chen, Yuanlin, Zhang zhou fu zhi [Crónica local de Zhangzhou], s.l., s.i., 1715.

Colín, Francisco, Labor evangélica, ministerios apostólicos de los obreros de la Compañía de Jesús, fundación y progresos de su provincia en las Islas Filipinas, t. II, Barcelona, Imprenta y Litografía de Henrich y Cía, 1900 [1. a ed. 1663].

Cruz, Gaspar da, Tratado das coisas de China (Évora, 1569-1570), Lisboa, Comissão Nacional para as Comemorações dos Descobrimentos Portugueses, 1997 [1. ${ }^{\mathrm{a}}$ ed. $\left.1569-1570\right]$.

Díaz-Trechuelo, Lourdes, Filipinas. La gran desconocida (1565-1898), Pamplona, Universidad de Navarra, 2001.

Escalante, Bernardino de, Discursos de Bernardino de Escalante al rey y sus ministros (1585-1605), edición de José Luis Casado Soto, Santander, Universidad de Cantabria, 1991 [1. a ed. 1577].

Fairbank, John King, China: Una nueva historia, Barcelona, Andrés Bello, 1997.

Fan, Shuzhi, Ming shi jiang gao [Lectura de la historia de Ming], Pekín, Compañía de Libros de Zhonghua, 2012.

Fang, Xing, «Lve lun ming chao wan li nian jian de "jin xian zhu gong" [Breve estudio sobre "Ofrecimiento para las obras" durante el reinado de Wanli]», Jiang xi she hui ke xue [Ciencias sociales de Jiangxi], 4, Nanchang, 2012, 108-113.

Feng, Chengjun, Zhu fang zhi jiao shi [Revisión y anotación de Zhu fan zhi], Pekín, Compañía del Libro de China, 2000 [1. ${ }^{\text {a }}$ ed. 1940].

Fish, Shirley, The Manila-Acapulco Galleons: The Treasure Ships of the Pacific, London, Author House, 2011.

Frank, Andre Gunder, Re-orientar: La economía global en la era del predominio asiático, Valencia, Universidad de Valencia, 2008.

García-Abásolo, Antonio, «La audiencia de Manila y los chinos de Filipinas. Casos de integración en el delito», en Fernández Árbol, José Luis Soberanes y Martínez de Codes, Rosa María (coords.), Homenaje a Alberto de la Hera, México D. F., UNAM, 2008, 339-368.

García-Abásolo, Antonio, «Los chinos y el modelo colonial español en Filipinas», Cuadernos de Historia Moderna, 10, Madrid, 2011, 223-242. 
García-Abásolo, Antonio, Murallas de piedra y cañones de seda: chinos en el imperio español (siglos XVI-XVIII), Córdoba, Universidad de Córdoba, 2012.

Gayo Aragón, Jesús, Ideas jurídico-teológicas de los religiosos de Filipinas en el Siglo XVI sobre la conquista de las Islas, Manila, Universidad de Santo Tomás, 1950.

Gernet, Jacques, El mundo chino, Barcelona, Crítica, 1999.

Gil, Juan, Los chinos en Manila (Siglos XVI y XVII), Lisboa, Centro Científico e Cultural de Macau, 2011.

Gorriz Abella, Jaume, Filipinas antes de Filipinas: El archipiélago de San Lázaro en el siglo XVI, Madrid, Polifemo, 2011.

Grocio, Hugo, Mare Liberum, sive de jure quod Batavis competit ad Indicana commercia dissertation, Leiden, Ludovici Elzevirii, 1609.

$\mathrm{Gu}$, Yanwu, Tian xia jun guo li bing shu [Libro de las ventajas y desventajas de todos los países del mundo], Shanghai, Librería de Shanghai, 1985.

Headley, J. M., «Spain's Asian Presence, 1565-1590: Structures and Aspirations», Hispanic American Historical Review, 75, State College, 1995, 623-645.

Huang, Renyu, Zhong guo da li shi [Gran Historia de China], Pekín, Librería de San Lian, 2007.

Huang, Zisheng y He, Sibing, Fei lv bin hua qiao shi [Historia de los inmigrantes chinos en las Filipinas], Guangzhou, Editorial de Educación Superior de Cantón, 2016.

Jordana y Morera, Ramón, La inmigración China en Filipinas, Madrid, Tipografía de Manuel G. Hernández, 1888.

Li, Chenguang, La estrategia china de Felipe II: la vía castellana (1556-1580), tesis doctoral dirigida por la doctora Elena Postigo Castellanos, Madrid, Universidad Autónoma de Madrid, 2018. Disponible en: https://repositorio.uam. es/handle/10486/683946 [consultado: 10/01/2021].

Liao, Dake y Hui, Ming, «16-18 shi ji chu ou zhou di tu zhong de Chincheo gang [El puerto de Chincheo en los mapas europeos desde el siglo XVI hasta el principio del siglo XVIII]», Zhong guo shi yan jiu [Investigación sobre la historia china], 1, Pekín, 2013, 159-176.

López-Gay, Jesús, «Ante la China del S. XVI, evangelización pacífica o conquista armada», Boletín de la Asociación Española de Orientalistas, 41, Madrid, 2005, 125-134.

Malo de Luque, Eduardo [seud. de Pedro de Góngora y Luján], Historia política de los establecimientos ultramarinos de las naciones europeos, t. V, Madrid, Antonio de Sancha, 1790.

Ming shi [Historia de Ming], 1739. Disponible en: «Zhong guo zhe xue shu dian zi hua ji hua (Plan de obras filosóficas electrónicas de China)», http://ctext.org/ wiki.pl?if=gb\&res=410835\&remap=gb [consultado: 10/01/2021].

Ming shi lu [Verdaderos registros de Ming], siglos XIV-XVII. Disponible en: «Zhong guo zhe xue shu dian zi hua ji hua (Plan de obras filosóficas 
electrónicas de China)», https://ctext.org/wiki.pl?if=gb\&res=964038\&remap $=\mathrm{gb}$ [consultado: 10/01/2021].

Morga, Antonio de, Sucesos de las Islas Filipinas, México D. F., Fondo de Cultura Económica, 2007 [1. ${ }^{a}$ ed. 1609].

Mote, Frederick W. y Twitchett, Denis (eds.), Jian qiao ming dai shi: 1368-1644 [Historia de la dinastía Ming de Cambridge: 1368-1644], vol. 1, Pekín, Editorial de Ciencias Sociales de China, 1992.

Ollé Rodríguez, Manel, La empresa de China. De la Armada Invencible al Galeón de Manila, Barcelona, El Acantilado, 2002.

Rojas, Juan Camilo, «Quejas y acusaciones por malas prácticas de gobierno contra Francisco de Sande, gobernador y Capitán General de las Islas Filipinas: 1575-1580», Historia y memoria, 19, Tunja, 2019, 25-65.

San Agustín, Gaspar de, Conquistas de las islas Filipinas (1565-1615), Madrid, Consejo Superior de Investigaciones Científicas, Instituto Enrique Flórez, 1975 [1. a ed. 1698].

Schirokauer, Conrad y Brown, Miranda, Breve historia de la civilización china, Barcelona, Bellaterra, 2006.

Song shi [Historia de Song], 1346. Disponible en «Zhong guo zhe xue shu dian zi hua ji hua (Plan de obras filosóficas electrónicas de China)», https://ctext.org/ wiki.pl?if=en\&res=975976 [consultado: 10/01/2021].

Spence, Jonathan D., En busca de la China moderna, Barcelona, Tusquets Editor, 2011.

Trigault, Nicolás, Istoria de la China i cristiana empresa hecha en ella por la Compañia de Iesus que, de los escritos del Padre Mateo Richo, compuso el Padre Nicolas Trigault flamenco, ambos de la misma Compañia; donde se descriven las costumbres, las leies i los estatutos de aquel Reino, i los dificultosissimos principios de su nueva iglesia; traduzida de lengua latina por el licenciado Duarte..., Sevilla, Gabriel Ramos Veiarano, 1621.

Vega y de Luque, Carlos Luis de, «Un proyecto utópico: la conquista de China por España», Boletín de la Asociación Española de Orientalistas, XV, Madrid, 1979, 45-69.

Vega y de Luque, Carlos Luis de, «Un proyecto utópico: la conquista de China por España», Boletín de la Asociación Española de Orientalistas, XVI, Madrid, 1980, 33-56.

Vega y de Luque, Carlos Luis de, «Un proyecto utópico: la conquista de China por España (Continuación)», Boletín de la Asociación Española de Orientalistas, XVII, Madrid, 1981, 3-38.

Vega y de Luque, Carlos Luis de, «Un proyecto utópico: la conquista de China por España (Conclusión)», Boletín de la Asociación Española de Orientalistas, XVIII:1, Madrid, 1982, 3-46.

Viforcos Marinas, María Isabel, «Carta e informe de Pedro de Valencia sobre los escritos del P. Alonso Sánchez y el Dr. Jerónimo Hurtado», en Valencia, Pedro de, Obras completas, VI, León, Universidad de León, 2012. 
Virgilio, La Eneida, Madrid, Edaf, 1985 [s. I a. C.].

Wan, Ming, «Ming dai bai yin huo bi hua: zhong guo yu shi jie lian jie de xin shi jiao [La monetización de la plata en la dinastía Ming: desde la nueva perspectiva de la conexión entre China y el mundo]», He bei xue kan [Revista Académica de Hebei], 3, Shijiazhuang, 2004, 145-154.

$\mathrm{Xu}$, Fuyuan, «Shu tong hai jin shu [Memorial para abolir la Prohibición del mar]», en Ming jing shi wen xian [Colección de los documentos a lo largo de la dinastía Ming], vol. 400, Pekín, Compañía del libro de Zhong Hua, 1962.

Yang, Li y Ye, Xiaodun, Dong nan ya de fu jian ren [Los pueblos de Fujian en Asia Sureste], Fuzhou, Editorial Popular de Fujian, 1993.

Zhang, Xie, Dong xi yang kao [Investigación sobre el océano oriental y occidental], Pekín, Compañía del libro de Zhong Hua, 1981 [1. ${ }^{a}$ ed. 1617].

Zhuang, Guotu y Chen, Huayue, Fei lv bin hua ren tong shi [Historia general de los chinos en las Filipinas], Xiamen, Universidad de Xiamen, 2012. 